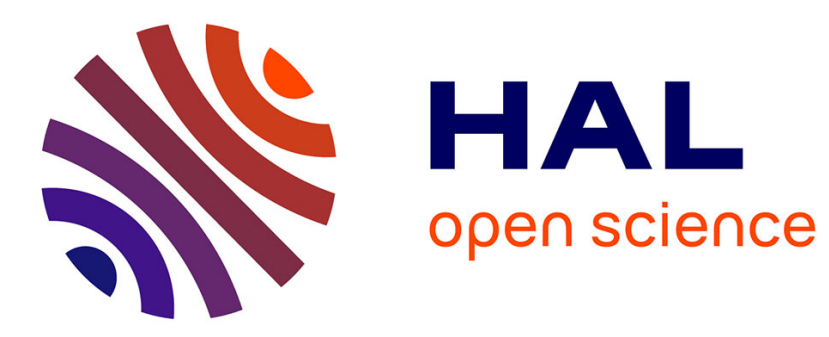

\title{
An ADMM Algorithm for Constrained Material Decomposition in Spectral CT
}

Tom Hohweiller, Nicolas Ducros, Françoise Peyrin, Bruno Sixou

\section{To cite this version:}

Tom Hohweiller, Nicolas Ducros, Françoise Peyrin, Bruno Sixou. An ADMM Algorithm for Constrained Material Decomposition in Spectral CT. EUSIPCO, Sep 2018, Rome, Italy. hal-01909643

\section{HAL Id: hal-01909643 \\ https://hal.science/hal-01909643}

Submitted on 31 Oct 2018

HAL is a multi-disciplinary open access archive for the deposit and dissemination of scientific research documents, whether they are published or not. The documents may come from teaching and research institutions in France or abroad, or from public or private research centers.
L'archive ouverte pluridisciplinaire HAL, est destinée au dépôt et à la diffusion de documents scientifiques de niveau recherche, publiés ou non, émanant des établissements d'enseignement et de recherche français ou étrangers, des laboratoires publics ou privés. 


\title{
An ADMM Algorithm for Constrained Material Decomposition in Spectral CT
}

\author{
Tom Hohweiller*, Nicolas Ducros*, Françoise Peyrin*†, Bruno Sixou* \\ * Univ. Lyon, INSA-Lyon, Université Claude Bernard Lyon 1, \\ UJM-Saint Etienne, CREATIS CNRS UMR 5220, Inserm U1206, F-69621, LYON, France \\ $\dagger^{\top}$ The European Synchrotron Radiation Facility, Grenoble, France
}

\begin{abstract}
Thanks to photon-counting detectors, spectral computerized tomography records energy-resolved data from which the chemical composition of a sample can be recovered. This problem, referred to as material decomposition, can be formulated as a nonlinear inverse problem. In previous work, we proposed to decompose the projection images using a regularized Gauss-Newton algorithm. To reduce further the ill-posedness of the problem, we propose here to consider equality and inequality constraints that are based on physical priors. In particular, we impose the positivity of the solutions as well the total mass in each projection image. In practice, we first decompose the projection images for each projection angle independently. Then, we reconstruct the sample slices from the decomposed projection images using a standard filtered-back projection algorithm. The constrained material decomposition problem is solved by the alternating direction method of multipliers (ADMM). We compare the proposed ADMM algorithm to the unconstrained GaussNewton algorithm in a numerical thorax phantom. Including constraints reduces the cross-talk between materials in both the decomposed projections and the reconstructed slices.

Index Terms-Alternating direction method of multipliers, spectral computed tomography, material decomposition, nonlinear inverse problem
\end{abstract}

\section{INTRODUCTION}

While standard computerized tomography (CT) integrates data over the X-ray spectrum, losing the energy signature of the materials, spectral CT (SPCT) keeps tracks of the energy of the detected photons. Therefore, the material composition of the sample can be recovered. There are various methods for acquiring energy-resolved data, which include the use of rapid $\mathrm{kVp}$-switching, multiple source beams, multi-layer detectors [1], or a photon-counting detector [2]. The latter approach records multiple energy bins using one source beam only, which reduces the irradiation dose. SPCT is particularly interesting for imaging K-edge materials [3] (i.e., gold, iodine or gadolinium) used as contrast agents and has many potential medical applications such as neck and head cancer [4].

Material decomposition and tomographic reconstruction can be done jointly [5] or sequentially, i.e., decomposing the projection images first and performing the tomographic reconstruction afterward [6], [7]. Here, we consider material

This work was supported by the grant ANR-11-INBS-0006 of the French National Research Agency (ANR) and was performed within the framework of the LABEX PRIMES (ANR-11-LABX-0063). It has also received funding from the European Union's Horizon 2020 research and innovation programme under the Marie Sklodowska-Curie grant agreement $\mathrm{N}^{\circ} 701915$. Contact: tom.hohweiller@creatis.insa-lyon.fr decomposition in the projection domain since it allows to decompose each projection in parallel. We propose to address material decomposition as a nonlinear inverse problem. In [7], we considered a Gauss-Newton algorithm to minimize a regularized cost function. This approach enjoys a fast convergence, but it does not ensure the solutions to be positive. Positivity constraints for material decomposition have been enforced in both the image [8] and projection [9] domains. However, these methods use first-order algorithm to minimize a cost function, thus with a low convergence rate. Our contribution here is to develop a fast constrained algorithm. In this work, we also consider equality constraints. Indeed, the quantity of injected contrast agent is known, which can improved material decomposition.

Our constrained algorithm uses the augmented Lagrangian formulation to incorporate inequality and equality constraints [10]-[14]. An alternating direction method of multipliers (ADMM) minimizes the Lagrangian function to recover the projected material maps. ADMM has been already used to postprocess volumes obtained afer tomographic reconstruction [15] or for joint decomposition-tomographic reconstruction [16]. Both approaches considered a linearized forward model. In this work, we consider a nonlinear forward model and perform material decomposition of the projections. Our constrained algorithm is tested on realistic simulated projections of numerical thorax phantom and compared with an unconstrained Gauss-Newton algorithm.

The rest of the paper is organized as follows. Fist, we present the forward and inverse problems. Second, we describe our optimization algorithm. Then, we report and discuss some numerical experiments.

\section{MATERIAL DECOMPOSITION IN SPECTRAL CT}

\section{A. Forward model}

Considering a photon-counting detector with $P$ pixels and $I$ energy bins, the measured data is gathered in a vector $\mathbf{s} \in$ $\mathbf{R}^{I P}$.

$$
\mathbf{s}=\left[s_{1,1}, \ldots, s_{I, 1}, \ldots, s_{I, P}\right]^{\mathrm{T}}
$$

The projected mass density describes the mass of $M$ materials on the $P$ pixels, thus $\mathbf{a} \in \mathbf{R}^{M P}$

$$
\mathbf{a}=\left[a_{1,1}, \ldots, a_{m, 1}, \ldots, a_{M, P}\right]^{\mathrm{T}}
$$


The standard forward model [6], [7] reads as:

$$
\mathbf{s}_{i, p}=\int_{\mathbf{R}} n_{i}^{0}(E) \exp \left(-\sum_{m=1}^{M} a_{m, p} \tau_{m}(E)\right) \mathrm{d} E
$$

where $\mathbf{s}_{i, p}$ is the number of photons detected at the pixel $p$ for the $i$ th energy bin, $n_{i}^{0}(E)$ is the effective spectrum (accounting for the incident spectrum and the detector response) at the energy $E$ for the $i$-th energy bin, $\tau_{m}(E)$ is a function representing the attenuation of the $m$-th material at the energy $E$ and $a_{m, p}$ is the projection mass of the $m$-th material at pixel $p$, such as

$$
a_{m, p}=\int_{\mathcal{L}_{p}} \rho_{m}(\mathbf{x}) \mathrm{d} \mathbf{x}
$$

with $\mathcal{L}_{p}$ is the $\mathrm{x}$-ray path and $\rho_{m}(\mathbf{x})$ is the material density at the voxel $\mathbf{x}$.

\section{B. Constrained Inverse problem}

For each projection angle, we propose to recover the material map a from the data $\mathbf{s}$ by solving the constrained optimization problem

$$
\min _{\mathbf{a}} \mathcal{C}(\mathbf{a}, \mathbf{s}) \quad \text { s.t }\left\{\begin{array}{l}
\mathbf{a} \geq 0 \\
\sum_{p} a_{m, p}=c_{m}
\end{array},\right.
$$

where $\mathcal{C}$ is the cost function and $c_{m}$ represents the total mass of the $m$-th material. Here, we assume that materials maps are positive and that the total mass of each material $c_{m}$ is know and constant across angles, which is a valid hypothesis when the detector field-of-view is wide enough.

To stabilize the minimization in the presence of noise, we consider

$$
\mathcal{C}(\mathbf{a}, \mathbf{s})=\mathcal{D}(\mathbf{a}, \mathbf{s})+\alpha_{\mathrm{R}} \mathcal{R}(\mathbf{a})
$$

where $\mathcal{D}$ is the data fidelity term, $\mathcal{R}$ the regularization term, and $\alpha_{\mathrm{R}}$ the regularization parameter. Following [7], we choose

$$
\mathcal{D}(\mathbf{a}, \mathbf{s})=\|\mathbf{s}-\mathcal{F}(\mathbf{a})\|_{\mathbf{W}}^{2}
$$

where $\mathcal{F}$ represents the forward model of Eq. (3) and $W$ is a diagonal weighting matrix with elements $1 / \sqrt{\mathrm{s}}$. Assuming that only three materials (soft tissues, bones and gadolinium as a marker) are present in the sample, we choose

$$
\mathcal{R}(\mathbf{a})=\left\|\Delta \mathbf{a}_{\text {soft }}\right\|_{2}^{2}+\left\|\nabla \mathbf{a}_{\text {bone }}\right\|_{1}+\left\|\nabla \mathbf{a}_{\mathrm{Gd}}\right\|_{1}
$$

where $\nabla$ and $\Delta$ are first- and second-order differential operator and $\|.\|_{2}$ and $\|.\|_{1}$ are the $\ell_{2}$ - and $\ell_{1}$-norm, respectively.

\section{COnstrained MATERIAL DECOMPOSITION ADMM ALGORITHM}

We propose an ADMM algorithm to solve the minimization problem described above. To this aim, we minimize the augmented Lagrangian functional

$$
\begin{aligned}
\mathcal{L}\left(\mathbf{a}, \mathbf{b}, \boldsymbol{\alpha}_{\mathrm{I}}, \alpha_{\mathrm{E}}, \mathbf{s}\right) & =\mathcal{D}(\mathbf{a}, \mathbf{s})+\alpha_{\mathrm{R}} \mathcal{R}(\mathbf{a}) \\
& +\mathcal{H}_{\mathrm{E}}\left(\mathbf{a}, \alpha_{\mathrm{E}}\right)+\mathcal{G}_{\mathrm{E}}(\mathbf{a}) \\
& +\mathcal{H}_{\mathrm{I}}\left(\mathbf{a}, \mathbf{b}, \boldsymbol{\alpha}_{\mathrm{I}}\right)+\mathcal{G}_{\mathrm{I}}(\mathbf{a}, \mathbf{b})+\mathbf{1}(\mathbf{b})
\end{aligned}
$$

where $\mathcal{H}_{\mathrm{E}}$ and $\mathcal{H}_{\mathrm{I}}$ are the Lagrangian terms associated to the equality and inequality constraints, respectively, $\mathcal{G}_{\mathrm{E}}$ and $\mathcal{G}_{\mathrm{I}}$ are the augmented Lagrangian terms, $\mathbf{1}$ denotes the indicator function of the positive orthant, $\alpha_{\mathrm{E}} \in \mathbf{R}$ and $\boldsymbol{\alpha}_{\mathrm{I}} \in \mathbf{R}^{M P}$ are the Lagrangian multipliers, and $\mathbf{b} \in \mathbf{R}^{M P}$ is an auxiliary variable.

1) Inequality constraint: To enforce the inequality constraints, we introduce an auxiliary variable $\mathbf{b}$ and rewrite the constraint as

$$
\begin{aligned}
& \mathbf{a}=\mathbf{b} \\
& \mathbf{b} \geq \mathbf{0}
\end{aligned}
$$

The constraints (10) and (11) translate into

$$
\begin{aligned}
\mathcal{H}_{\mathrm{I}}\left(\mathbf{a}, \mathbf{b}, \boldsymbol{\alpha}_{\mathrm{I}}\right) & =\boldsymbol{\alpha}_{\mathrm{I}}^{\mathrm{T}}(\mathbf{b}-\mathbf{a}) \\
\mathcal{G}_{\mathrm{I}}(\mathbf{a}, \mathbf{b}) & =\frac{\beta_{\mathrm{I}}}{2}\|\mathbf{b}-\mathbf{a}\|_{2}^{2}
\end{aligned}
$$

whereas Eq. (11) is formulated with the term

$$
\mathbf{1}(\mathbf{b})= \begin{cases}0, & \text { if } \mathbf{b} \geq 0 \\ \infty, & \text { otherwise }\end{cases}
$$

Where $\beta_{\mathrm{I}} \in \mathbf{R}$ is an augmented Lagrangian parameter.

2) Equality constraint: The equality constraints lead to

$$
\begin{aligned}
\mathcal{H}_{\mathrm{E}}\left(\mathbf{a}, \alpha_{\mathrm{E}}\right) & =\alpha_{\mathrm{E}}\left(\sum_{p=1}^{P} \frac{a_{\mathrm{gd}, p}}{c_{\mathrm{gd}}}-1\right) \\
\mathcal{G}_{\mathrm{E}}(\mathbf{a}) & =\frac{\beta_{\mathrm{E}}}{2}\left(\sum_{p=1}^{P} \frac{a_{\mathrm{gd}, p}}{c_{\mathrm{gd}}}-1\right)^{2}
\end{aligned}
$$

where $\beta_{\mathrm{E}} \in \mathbf{R}$ is an augmented Lagrangian parameter. Note that the constraint applies to the gadolinium material only.

3) Minimization scheme: According to the ADMM framework, a saddle point of the augmented Lagrangian functional is obtained alternating the update of the primal variables and the Lagrangian multipliers [10]:

$$
\begin{aligned}
& \mathbf{a}^{\ell+1} \in \underset{\mathbf{a}}{\operatorname{argmin}} \mathcal{L}\left(\mathbf{a}, \mathbf{b}^{\ell}, \alpha_{\mathrm{E}}^{\ell}, \boldsymbol{\alpha}_{\mathrm{I}}^{\ell}\right) \\
& \mathbf{b}^{\ell+1} \in \underset{\mathbf{b}}{\operatorname{argmin}} \mathcal{L}\left(\mathbf{a}^{\ell+1}, \mathbf{b}, \alpha_{\mathrm{E}}^{\ell}, \boldsymbol{\alpha}_{\mathrm{I}}^{\ell}\right) \\
& \alpha_{\mathrm{E}}^{\ell+1} \in \underset{\alpha_{\mathrm{E}}}{\operatorname{argmax}} \mathcal{L}\left(\mathbf{a}^{\ell+1}, \mathbf{b}^{\ell+1}, \alpha_{\mathrm{E}}, \boldsymbol{\alpha}_{\mathrm{I}}^{\ell}\right) \\
& \boldsymbol{\alpha}_{\mathrm{I}}^{\ell+1} \in \underset{\boldsymbol{\alpha}_{\mathrm{I}}}{\operatorname{argmax}} \mathcal{L}\left(\mathbf{a}^{\ell+1}, \mathbf{b}^{\ell+1}, \alpha_{\mathrm{E}}^{\ell+1}, \boldsymbol{\alpha}_{\mathrm{I}}\right)
\end{aligned}
$$

The projected mass map a is updated using a Gauss-Newton algorithm (SPRAY toolbox [17]), the auxiliary variable $\mathbf{b}$ is updated using a proximal algorithm, and the Lagrangian multipliers $\alpha_{\mathrm{E}}$ and $\boldsymbol{\alpha}_{\mathrm{I}}$ are updated with an ascent gradient algorithm [10]. The parameters $\beta_{\mathrm{E}}$ and $\beta_{\mathrm{I}}$ are increased every outer iteration $\ell$ with a multiplicative udpate:

$$
\begin{aligned}
& \beta_{\mathrm{E}}^{\ell+1}=\omega \beta_{\mathrm{E}}^{\ell} \\
& \beta_{\mathrm{I}}^{\ell+1}=\omega \beta_{\mathrm{I}}^{\ell}
\end{aligned}
$$

with a positive constant $\omega>1$. This update rule for the Lagrangian parameter allows the algorithm to minimize in 


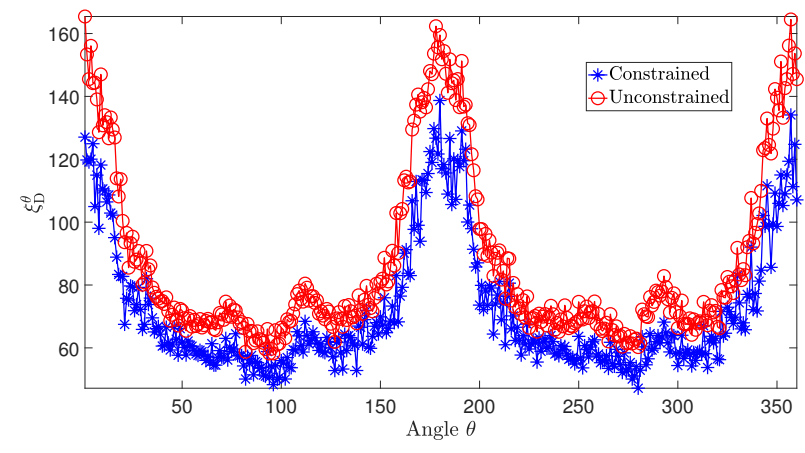

Fig. 1. Euclidean decomposition error $\xi_{\mathrm{D}}^{\theta}$ over all projections for the constrained algorithm (in blue) and the unconstrained algorithm (in red)

the first iterations the function $\mathcal{C}(\mathbf{a}, \mathbf{s})$, and then after a few outer iterations the constraints since they become the predominant contribution to the Lagrangian. Denoting $\Pi$ the projector operation onto the positive orthant and considering elements-wise operations, the constrained algorithm is given in Algorithm 1.

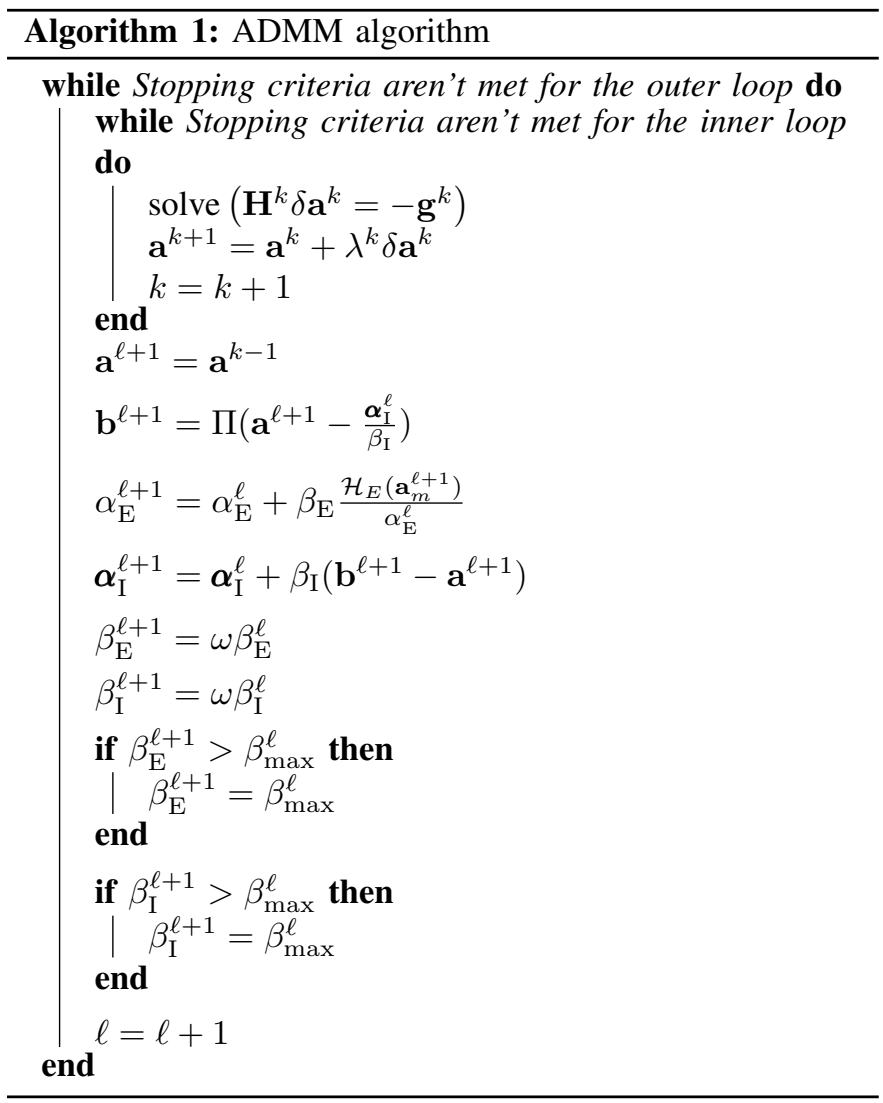

\section{NUMERICAL EXPERIMENTS}

\section{A. Phantom, simulation and decomposition parameters}

In this work, a numerical phantom of a thorax is considered $(240 \times 185 \times 84$ voxels $)$ [18]. The phantom is composed of $M=3$ materials, namely soft tissues, bones and gadolinium (in the portal vein). Projections are created using the radon function of Matlab, providing $84 \times 306$ images.

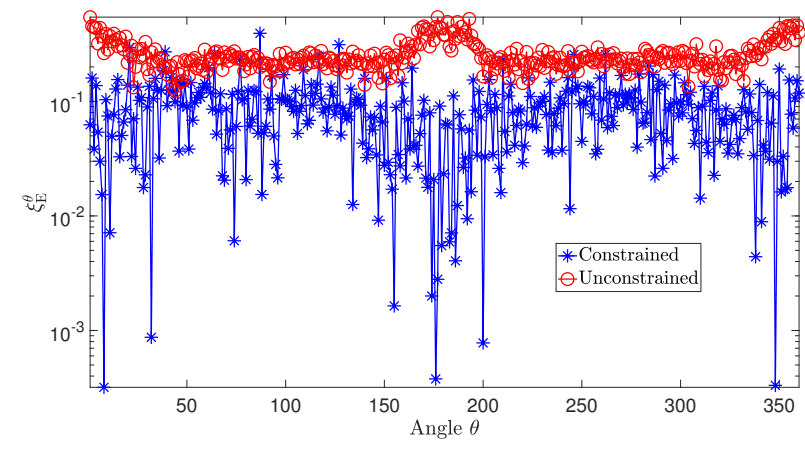

Fig. 2. Quantification error $\xi_{\mathrm{E}}^{\theta}$ over all projections for the constrained algorithm (in blue) and the unconstrained algorithm (in red)

We consider a $120 \mathrm{kV}$ X-Ray source, generated by the spekCalc software [19]. The photon-counting detector detect photons in $I=4$ energy bins. For each pixel, $10^{7}$ photons are send.

For both algorithms (unconstrained and constrained), the initialization is the same, with uniform materials maps $\mathbf{a}_{m}^{0}=0$ g. $\mathrm{cm}^{-2}$ for all materials. The regularization parameter is the same as well, set at $\alpha_{\mathrm{R}}=1$. For the unconstrained algorithm, the algorithm stops after 100 iterations to ensure the optimal solution, for a better comparison. For the ADMM algorithm the Lagrangian multipliers are initialized $\alpha_{\mathrm{E}}^{0}=0$ and $\boldsymbol{\alpha}_{\mathrm{I}}^{0}=\mathbf{0}$. The following initialisation parameters are used: $\beta_{\mathrm{E}}^{0}=10^{0}$ and $\beta_{\mathrm{I}}^{0}=10^{-2}$, with a maximum at $\beta_{\max }^{\ell}=10^{10}$ and an evolution parameter $\omega=1.5$. The algorithm stops the inner loop (update of a, Eq. (17)) if the relative decrease of $\mathcal{L}$ is small $\left(<10^{-3}\right)$ or if there is more than 30 iterations. The outer loop stops if the constraints are satisfied: $\left\|\mathbf{a}^{\ell}-\mathbf{b}^{\ell}\right\|<$ $10^{-3}$ and $\left|\left(\sum_{p}^{P} \frac{a_{\mathrm{gd}, \mathrm{p}}^{\ell}}{c_{\mathrm{gd}}}-1\right)\right|<10^{-3}$ for the index $m=$ corresponding to gadolinium.

The decomposition are made for every degree in the range $\theta=\left[0^{\circ}, 359^{\circ}\right]$. We denote $\mathbf{a}^{\theta}$ the decomposition for the angle $\theta$ and $\boldsymbol{\rho}^{z}$ the decomposed material at a given slice $z$.

\section{B. Evaluation of the methods}

The constraints are evaluated by looking at the percentage of negative values, the smallest value in the projections: $\min \left(\mathbf{a}_{m}^{\theta}\right) \forall m, \theta$ and by computing the relative distance between the sum of the gadolinium projections and the constraint $c_{\text {gd }}$ :

$$
\xi_{\mathrm{E}}^{\theta}=\frac{\left|\sum_{p=1}^{P} a_{\mathrm{gd}, \mathrm{p}}^{\theta}-c_{\mathrm{gd}}\right|}{c_{\mathrm{gd}}}
$$

The decomposed projections are also evaluated by computing the $\ell_{2}$ distance between the decomposition and the ground truth:

$$
\xi_{\mathrm{D}}^{\theta}=\sum_{m}^{M}\left\|\mathbf{a}_{m}^{\theta}-\mathbf{a}_{m}^{\text {truth }, \theta}\right\|_{2}
$$

In the image domain (after the tomographic reconstruction), the $\ell_{2}$ distance is also computed to evaluate the decomposed 

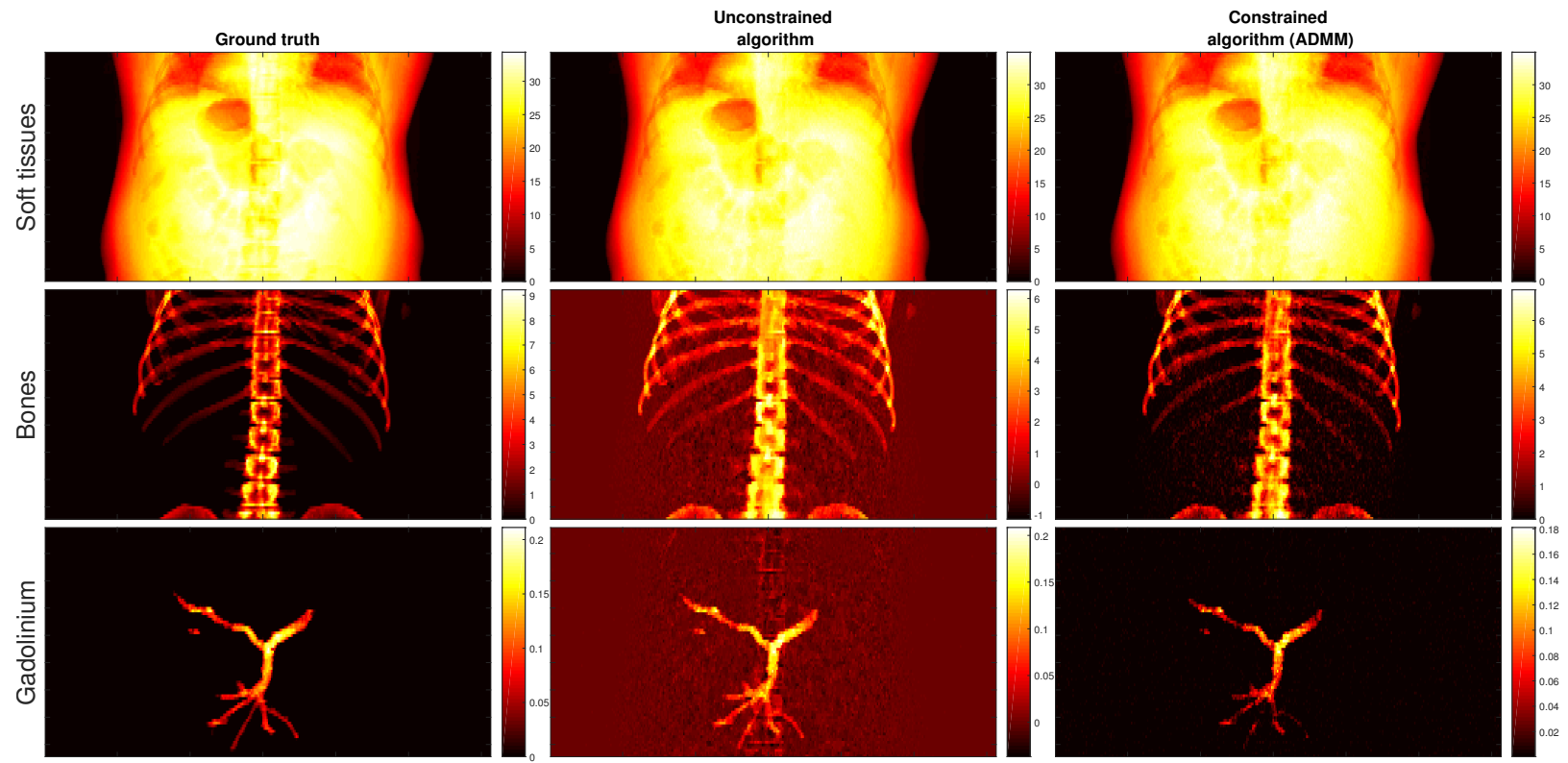

Fig. 3. Decomposition of the thorax for $\theta=90^{\circ}$. Ground truth (first column), unconstrained algorithm (second column) and constrained (ADMM) algorithm (third column) for the 3 different materials: soft tissues (top row), bones (middle row) and gadolinium (bottom row).

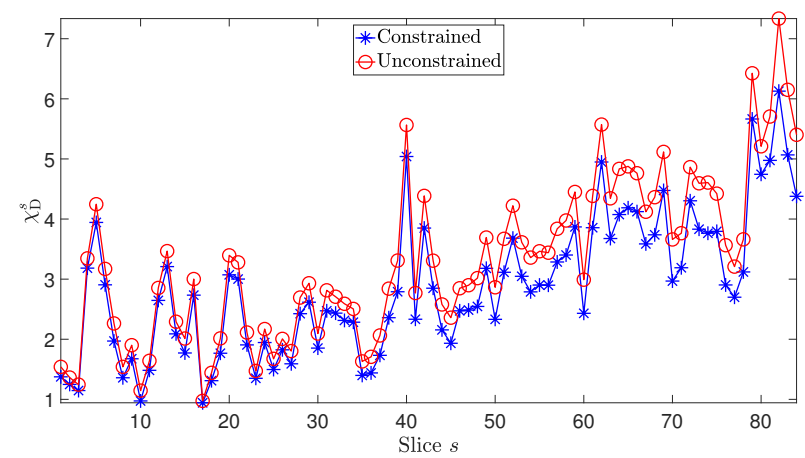

Fig. 4. Euclidiean decomposition error $\chi_{\mathrm{D}}^{s}$ over all slices for the constrained algorithm (in blue) and the unconstrained algorithm (in red)

slices, as:

$$
\chi_{\mathrm{D}}^{z}=\sum_{m}^{M}\left\|\boldsymbol{\rho}_{m}^{z}-\boldsymbol{\rho}_{m}^{\text {truth }, z}\right\|_{2}
$$

where $\boldsymbol{\rho}_{m}^{\text {truth, } z}$ is the reconstructed slice from the ground truth sinogram.

\section{RESULTS AND DISCUSSION}

\section{A. Projection domain}

Table I presents the percentage of negative values, the smallest projection value, and the smallest and highest values for $\xi_{\mathrm{E}}$ for all the angles $\theta$. The error $\xi_{\mathrm{D}}^{\theta}$ and $\xi_{\mathrm{E}}^{\theta}$ are displayed in Fig. 1 and Fig. 2, respectively, for all angles $\theta$. These metrics show that the constrained algorithm gives better decomposition than the unconstrained one (by looking at the error $\xi_{\mathrm{D}}$ ), and that the constraints are well satisfied for the constrained algorithm. The decomposition images are displayed in Fig. 3 for $\theta=90^{\circ}$. The first column displays the ground truth, the

\begin{tabular}{|c|c|c|}
\cline { 2 - 3 } \multicolumn{1}{c|}{} & Constrained algorithm & Unconstrained algorithm \\
\hline \% of negative value & $\mathbf{2 . 0 8} \%$ & $27.7 \%$ \\
\hline Smallest value & $\mathbf{- 0 . 0 2}$ & -3.78 \\
\hline Smallest $\xi_{\mathrm{E}}$ & $\mathbf{3 . 1 9} \times \mathbf{1 0}^{-4}$ & 0.12 \\
\hline Biggest $\xi_{\mathrm{E}}$ & $\mathbf{0 . 3 9}$ & 0.54 \\
\hline
\end{tabular}

TABLE I

Percentage of Negative VALUES, SMALlEST VALUE, SMALLEST AND LARGEST $\xi_{E}$, CONSIDERING ALL PROJECTIONS FOR BOTH ALGORITHMS.

second one the reconstruction obtained with the unconstrained algorithm and the third one the image obtained with the constrained algorithm (ADMM). We can see that the proposed algorithm gives visually better decomposition. Moreover, there is less bones in the gadolinium image, i.e., less cross-talk.

\section{B. Image domain}

The evolution of the error $\chi_{\mathrm{D}}$ is displayed on Fig. 4 as a function of the slice number $z$. As in the projection domain, a better decomposition is obtained with the proposed constrained algorithm. Fig. 5 displays two slices: $z=1$ and $z=40$. The first column shows the ground truth, the second one the slice reconstructed with unconstrained algorithm and the third one the reconstruction obtained with the constrained algorithm. The left picture, for $z=1$, is a slice where there is no marker. The constrained algorithm leads to very small values whereas the unconstrained one shows some bone regions. The effect of the constrained algorithm is more clear at slice $z=40$ where there is a lot of cross-talk with the unconstrained algorithm that disappears with our algorithm.

\section{Conclusion}

We proposed an algorithm for material decomposition in SPCT. The non linear inverse ill-posed problem is solved by minimizing a cost function. Equality and inequality constraints 

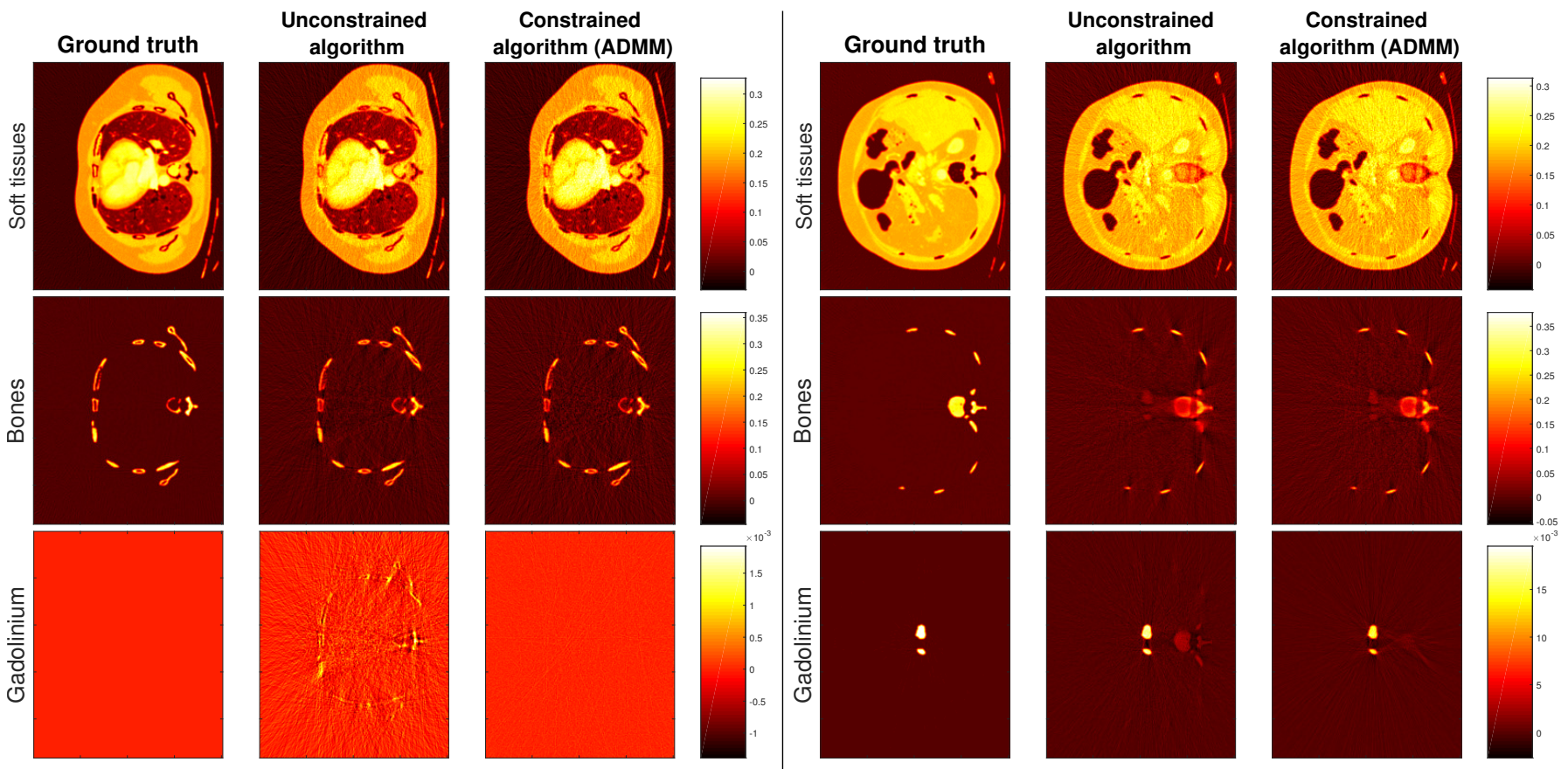

Fig. 5. Reconstruted slice of the thorax after tomographic reconstruction for $z=1$ (left) and $z=40$ (right). Ground truth (first column), unconstrained algorithm (second column) and constrained (ADMM) algorithm (third column) for the 3 different materials: soft tissues (top row), bones (middle row) and gadolinium (bottom row), for each slice.

were included in an augmented Lagrangian formulation. An ADMM algorithm was used to found its saddle point. Our new algorithm was compared with an unconstrained algorithm (a Gauss-Newton algorithm) on a numerical phantom of a thorax. Decomposition on the projections shows an improvement (qualitatively and quantitatively) of the material decomposition. Indeed, the cross-talk is reduced when the constraints are included. The improvement of the decomposition on the projection domain is maintained in the image domain after the tomographic reconstruction. Future work will address the decomposition of real data.

\section{REFERENCES}

[1] T. R. Johnson, "Dual-energy ct: general principles," American Journal of Roentgenology, vol. 199, no. 5_supplement, pp. S3-S8, 2012.

[2] K. Taguchi and J. Iwanczyk, "Vision 20/20: Single photon counting x-ray detectors in medical imaging," Med. Phys, vol. 40, no. 10, 2013.

[3] D. P. Cormode, E. Roessl, A. Thran, T. Skajaa, R. E. Gordon, J.-P. Schlomka, V. Fuster, E. A. Fisher, W. J. Mulder, R. Proksa et al., "Atherosclerotic plaque composition: analysis with multicolor ct and targeted gold nanoparticles 1," Radiology, vol. 256, no. 3, pp. 774-782, 2010.

[4] A. Pérez-Lara and R. Forghani, "Spectral computed tomography: Technique and applications for head and neck cancer," Magnetic Resonance Imaging Clinics, vol. 26, no. 1, pp. 1-17, 2018.

[5] Y. Long and J. A. Fessler, "Multi-material decomposition using statistical image reconstruction for spectral ct," IEEE transactions on medical imaging, vol. 33, no. 8, pp. 1614-1626, 2014.

[6] J. Schlomka and et al, "Experimental feasibility of multi-energy photoncounting k-edge imaging in pre-clinical computed tomography," $P M B$, vol. 53, no. 15 , p. 4031, 2008.

[7] N. Ducros, J. Abascal, B. Sixou, S. Rit, and F. Peyrin, "Regularization of nonlinear decomposition of spectral x-ray projection images," Med. Phys, vol. 44, no. 9, 2017.
[8] E. Y. Sidky and X. Pan, "Image reconstruction in circular cone-beam computed tomography by constrained, total-variation minimization," Physics in Medicine \& Biology, vol. 53, no. 17, p. 4777, 2008.

[9] J. Noh, J. A. Fessler, and P. E. Kinahan, "Statistical sinogram restoration in dual-energy ct for pet attenuation correction," IEEE transactions on medical imaging, vol. 28, no. 11, pp. 1688-1702, 2009.

[10] J. Eckstein and W. Yao, "Augmented lagrangian and alternating direction methods for convex optimization: A tutorial and some illustrative computational results," RUTCOR Research Reports, vol. 32, p. 3, 2012.

[11] S. Wright and J. Nocedal, "Numerical optimization," Springer Science, vol. 35 , no. $67-68$, p. $7,1999$.

[12] M. V. Afonso, J. M. Bioucas-Dias, and M. A. Figueiredo, "Fast image recovery using variable splitting and constrained optimization," IEEE transactions on image processing, vol. 19, no. 9, pp. 2345-2356, 2010.

[13] _ _ "An augmented lagrangian approach to the constrained optimization formulation of imaging inverse problems," IEEE Transactions on Image Processing, vol. 20, no. 3, pp. 681-695, 2011.

[14] M. K. Ng, P. Weiss, and X. Yuan, "Solving constrained total-variation image restoration and reconstruction problems via alternating direction methods," SIAM journal on Scientific Computing, vol. 32, no. 5, pp. 2710-2736, 2010.

[15] Q. Ding, T. Niu, X. Zhang, and Y. Long, "Image-domain multi-material decomposition for dual-energy ct based on correlation and sparsity of material images," arXiv preprint arXiv:1710.07028, 2017.

[16] — " "Image-domain multi-material decomposition for dual-energy ct via total nuclear norm and $\ell_{0}$ norm."

[17] "Spray toolbox," lyon.fr/ ducros/WebPage/spray.html.

https://www.creatis.insa-

[18] R. Kéchichian, S. Valette, M. Desvignes, and R. Prost, "Shortest-path constraints for $3 \mathrm{~d}$ multiobject semiautomatic segmentation via clustering and graph cut," IEEE TIP, vol. 22, no. 11, pp. 4224-4236, 2013.

[19] G. Poludniowski, G. Landry, F. DeBlois, P. Evans, and F. Verhaegen, "Spekcalc: a program to calculate photon spectra from tungsten anode x-ray tubes," $P M B$, vol. 54, no. 19, 2009. 